\title{
Hepatocellular Carcinoma with Segmental Portal Vein Invasion Exhibiting a Complete Response after Transarterial Radioembolization
}

\author{
Jun Sik Yoon ${ }^{1,2}$, Su Jong Yu', Yun Bin Lee', Eun Ju Cho', Jeong-Hoon Lee', Yoon Jun Kim', Jung-Hwan Yoon \\ 'Department of Internal Medicine and Liver Research Institute, Seoul National University College of Medicine, Seoul; ${ }^{2}$ Department of \\ Internal Medicine, Busan Paik Hospital, Inje University College of Medicine, Busan, Korea
}

Received Jul. 31, 2019

Revised Aug. 17, 2019

Accepted Aug. 22, 2019
The treatment options available for patients with hepatocellular carcinoma (HCC) with portal vein invasion (PVI) include sorafenib, transarterial radioembolization (TARE), radiation therapy (RT), transarterial chemoembolization with $\mathrm{RT}$, and proton beam irradiation. Herein, we present a case of HCC with segmental PVI that was managed via TARE. The patient had a $4 \mathrm{~cm}$ HCC that invaded the segment VIII portal vein branch without extrahepatic spread. Liver function was Child-Pugh grade A, and performance status was good. TARE was performed without any adverse events, and a radiological complete response (CR) was achieved. Thereafter, the patient was followed-up every 3-6 months without any further treatment, and the $\mathrm{CR}$ was maintained for $>3$ years. Therefore, TARE may be a useful alternative therapeutic option for patients with HCC exhibiting segmental PVI. (J Liver Cancer 2019;19:159-164)

Keywords: Hepatocellular carcinoma; Venous thrombosis; Radiotherapy; Therapeutic embolization; Yttrium-90

\section{INTRODUCTION}

Hepatocellular carcinoma (HCC) with portal vein invasion (PVI) is classified as an advanced stage tumor according to the Barcelona Clinic Liver Cancer (BCLC) staging system; sorafenib is the first-line therapeutic option for patients with this disease. ${ }^{1}$ However, sorafenib resulted in only a modest survival benefit compared to placebos in such HCC patients. ${ }^{2-4}$ Alternative therapeutic options include transarterial radioembolization (TARE), stereotactic body radiation therapy (RT), transarterial chemoembolization (TACE) plus RT, and proton beam irradiation; all have been used to treat pa-

\section{Corresponding author: Su Jong Yu}

Department of Internal Medicine, Seoul National University Hospital, 101 Daehak-ro, Jongno-gu, Seoul 03080, Korea

Tel. +82-2-6072-7216, Fax. +82-2-743-6701

E-mail; ydoctor2@hanmail.net

https://orcid.org/0000-0001-8888-7977 tients with advanced HCC without extrahepatic metastases. In a recent study, TACE plus RT exhibited a significant survival benefit compared with sorafenib for patients with advanced HCC accompanied by macroscopic vascular invasion. ${ }^{5}$ However, the current practice guidelines indicate that patients with advanced HCC accompanied by macroscopic vascular invasion are contra-indicated for TACE because of the high risks of post-embolization syndrome and hepatic insufficiency. ${ }^{6-8}$ Accordingly, TARE is a useful option for patients with HCC with PVI; TARE is associated with a longer time to progression, a lower incidence of post-embolization syndrome, and a shorter hospitalization than those observed after TACE. ${ }^{9-12}$ Herein, we report a case of HCC with segmental PVI that was successfully managed with TARE. 


\section{CASE REPORT}

\section{Clinical findings}

A 50-year-old man visited our outpatient clinic for the treatment of a single hepatic mass detected on computed tomography (CT) performed during surveillance at another hospital. The patient did not have any associated symptoms. He had been diagnosed with hepatitis $B$ virus-related liver cirrhosis (LC) and was being treated with entecavir. He was regularly followed-up for surveillance of HCC. Until detection of the hepatic mass, the patient had never experienced any complications associated with LC, such as ascites, hepatic encephalopathy, varices, or HCC. Physical examination did not reveal any abnormal findings, and the patient appeared healthy. The initial complete blood cell count panel revealed that the white blood cell count was $4.3 \times 10^{6} / \mathrm{L}$, the hemoglobin level was $16.8 \mathrm{~g} / \mathrm{dL}$, and the platelet count was $152 \times 10^{6} / \mathrm{L}$. A liver function test revealed a serum bilirubin level of $1.0 \mathrm{mg} / \mathrm{dL}$, an albumin level of $4.5 \mathrm{~g} / \mathrm{dL}$, a prothrombin time international normalized ratio of 1.03, an aspartate aminotransferase level of $25 \mathrm{IU} / \mathrm{L}$, and an alanine aminotransferase level of 29 IU/L. The serum level of alpha-fetoprotein (AFP) was $25,700 \mathrm{ng} / \mathrm{mL}$, and that of protein induced by vitamin $\mathrm{K}$ absence-II (PIVKA-II) was $51 \mathrm{mAU} / \mathrm{mL}$.

\section{Imaging findings}

An initial abdominal dynamic CT scan revealed a cirrhotic liver and a $4-\mathrm{cm}$ hepatic mass in segment VII/VIII, without any intrahepatic metastasis or lymph node enlargement. The hepatic mass exhibited typical HCC features of early arterial enhancement and portal venous washout (Fig. 1). Dynamic magnetic resonance imaging (MRI) was performed to further evaluate the extent of HCC; the segment VIII portal vein branch was involved (Fig. 2). Neither positron emission tomography-CT nor chest CT revealed any extrahepatic tumor metastasis.

\section{Diagnosis and treatment}

On the basis of the clinical and imaging findings, the patient was diagnosed with a 4-cm HCC invading the segment VIII portal vein branch without any extrahepatic spread. He had a preserved liver function (Child-Pugh score of 5) and a performance status score of 0 . Therefore, the HCC was classified as an advanced stage tumor by using the BCLC staging system, and as a stage III (T3, N0, M0) tumor according to the modified International Union Against Cancer system. We decided to perform TARE using the radioisotope yttrium $90\left({ }^{90} \mathrm{Y}\right)$. Hepatic angiography and ${ }^{99 \mathrm{~m}}$ technecium-macroaggregated albumin $\left({ }^{99 \mathrm{~m}} \mathrm{Tc}-\mathrm{MAA}\right)$ nuclear scan were performed as pretreatment simulation tests. Hepatic angiography revealed a hypervascular tumor in segments VII/VIII but without any anatomical variation of the hepatic arterial system (Fig. 3). On the ${ }^{99 \mathrm{~m}}$ Tc-MAA nuclear scan, the lung shunt fraction was 5\%; the patient was thus eligible for TARE without any dose reduction. TARE was performed by using TheraSphere ${ }^{\circledR}$ glass microspheres (BTG, London, UK); the total infused radiation activity was $3.95 \mathrm{GBq}$. The dose was calcu-
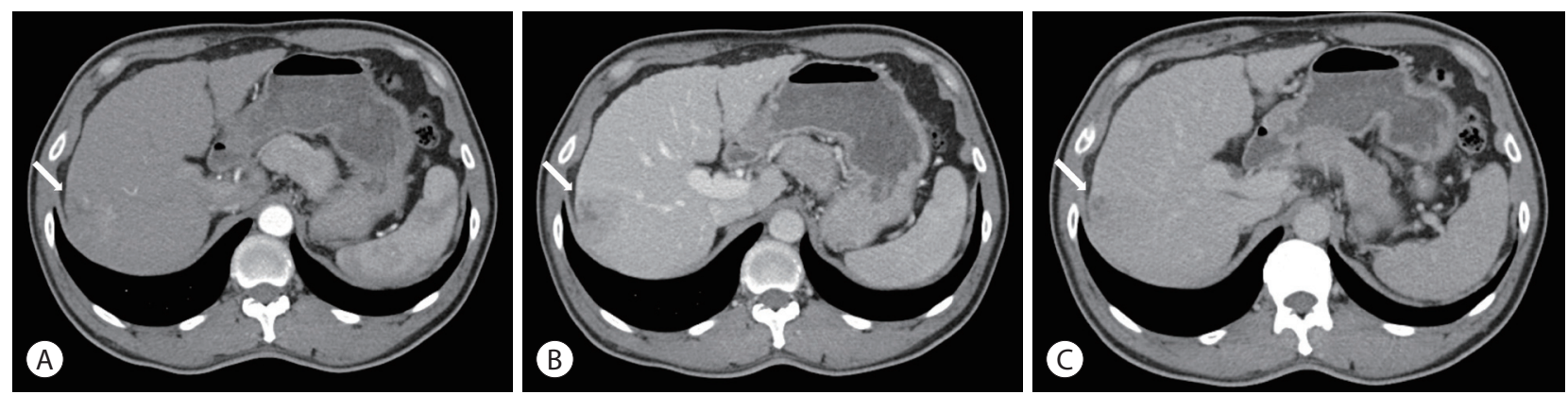

Figure 1. Initial dynamic computed tomography findings. A single $4 \mathrm{~cm}$ mass in segments VII/VIII exhibited enhancement in the arterial phase (A), and washout in the portal-phase (B) and the delayed phase (C) (arrow). 

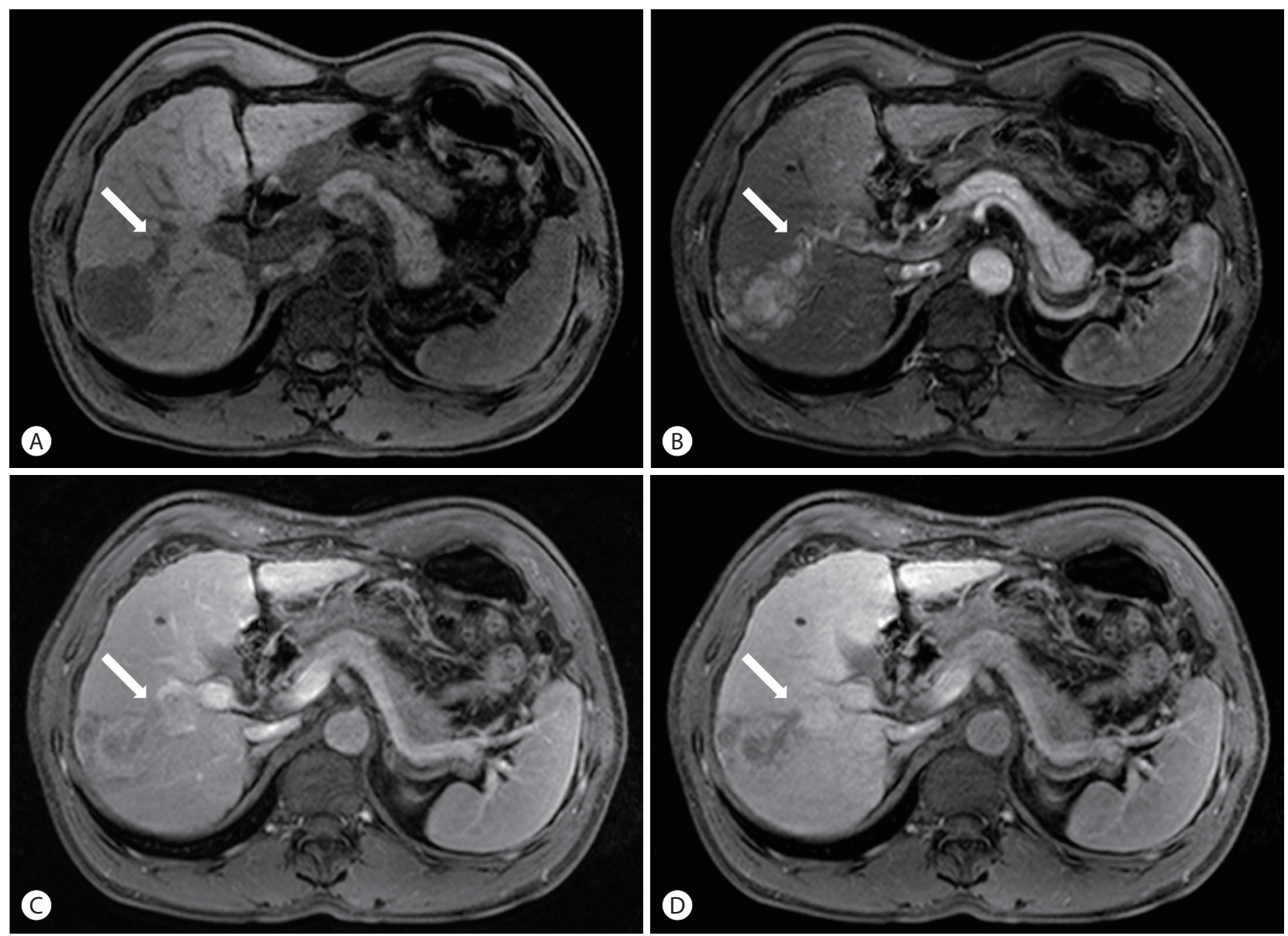

Figure 2. Magnetic resonance imaging findings. The mass showed low signal intensity on unenhanced T1-weighted imaging (A). In the dynamic phase, the mass exhibited enhancement in the arterial phase (B), and washout in the portal-phase (C) and the hepatobiliary phase (D). The tumor thrombus involved the segment VIII portal vein branch (arrow). The thrombus extended directly from the tumor and exhibited enhancement in the arterial phase, and washout in the portal- and hepatobiliary phases.

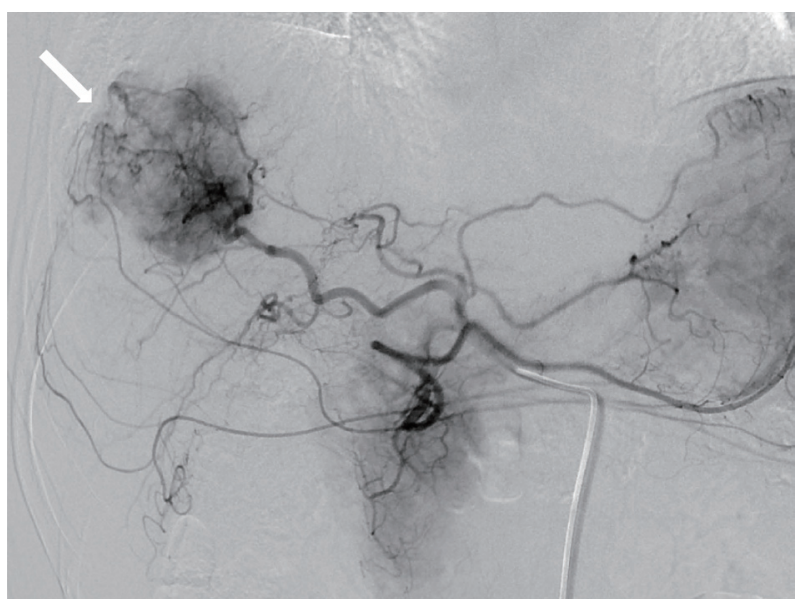

Figure 3. Hepatic angiography findings. Hepatic angiography revealed a hypervascular tumor in segments VII/VIII (arrow). The anatomy of the hepatic artery was normal; extrahepatic accessory arteries were absent. lated using the Medical Internal Radiation Dose method, as recommended by the microsphere manufacturer. No adverse events or clinical signs of liver function deterioration were noted after the procedure. After 2 months, the tumor response was evaluated according to the modified Response Evaluation Criteria in Solid Tumors, ${ }^{13}$ and a complete response (CR) was identified. The serum levels of AFP and PIVKA-II decreased from 25,700 to $76.5 \mathrm{ng} / \mathrm{mL}$ and from 51 to $14 \mathrm{mAU} / \mathrm{mL}$, respectively. The serum levels of the tumor markers dropped to within the normal ranges 3 months after TARE. The patient was followed-up every 3-6 months after the initial tumor response evaluation, and radiological CR was maintained for $>3$ years (Fig. 4). 

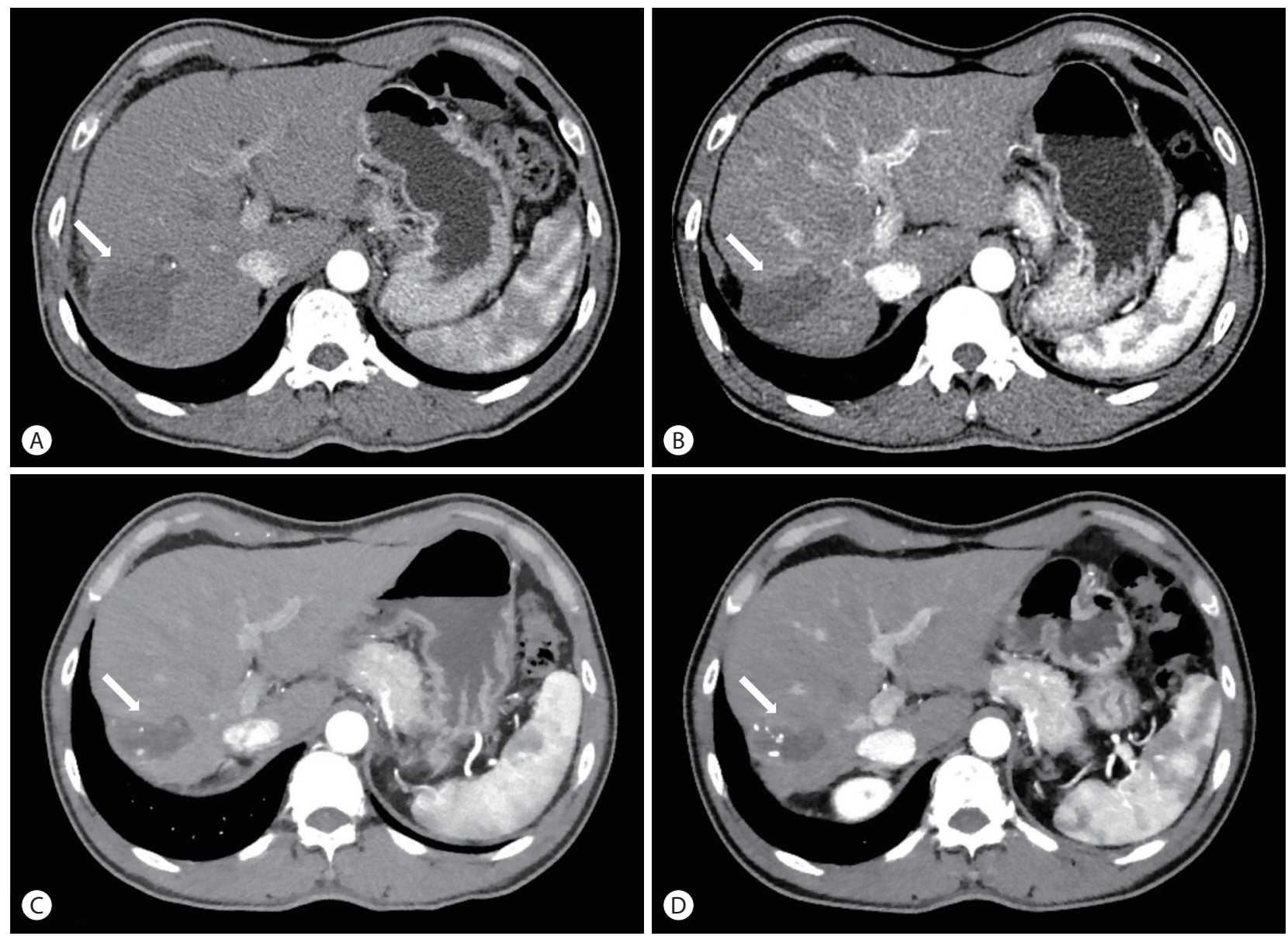

Figure 4. Findings on follow-up dynamic computed tomography after transarterial radioembolization (TARE). Low-attenuated necrotic change without any enhancement (a complete response [CR]) was observed at the tumor site two months after TARE (A). The necrotic lesion gradually decreased in size, and the CR status was maintained for 1 year (B), 2 years (C), and 3 years (D) after TARE (arrow).

\section{DISCUSSION}

In this study, we present a case of HCC treated with TARE. PVI was not identified on initial dynamic CT. However, because of markedly elevated serum level of AFP $(25,700 \mathrm{ng} / \mathrm{mL})$, MRI was performed to evaluate the presence of hidden vascular invasion or intrahepatic metastasis. On dynamic MRI by using Primovist ${ }^{\circledR}$, we identified the involvement of the segment VIII portal vein branch. Because the patient exhibited preserved liver function and a small tumor burden, we performed TARE rather than systemic therapy as the initial treatment. TARE was successfully performed without any adverse events or liver function deterioration. The patient exhibited a CR after TARE. In patients with advanced HCC, the median overall survival was as short as 12.3-13.6 months even after systemic therapy with sorafenib or lenvatinib. ${ }^{14}$ However, the current patient maintained a CR (i.e., did not experience relapse) for $>3$ years after TARE, which may therefore be an excellent treatment option for patients with HCC with segmental PVI.

According to current practice guidelines, systemic sorafenib or lenvatinib is the first-line therapeutic option for advanced HCC invading the PVI. ${ }^{7,8}$ However, the expected survival gain is only $2-3$ months, ${ }^{3,4}$ which was inadequate considering that the current patient had preserved liver function and only a small tumor burden. Surgical resection demonstrated substantial survival benefit (median overall survival of 22 months) in patients with segmental PVI. ${ }^{15}$ However, we did not consider surgical resection as a treatment option owing to the high serum AFP level $(25,700 \mathrm{ng} / \mathrm{mL})$, because a 
high serum AFP level ( $\geq 1,000 \mathrm{ng} / \mathrm{mL}$ ) is a major predictor of poor survival after surgical resection in patients with HCC. ${ }^{16}$ Therefore, we considered locoregional therapy such as TARE or TACE plus RT as the initial treatment. On the basis of the PVI status of the patient, we selected TARE rather than TACE plus RT because TARE is associated with a lower risk of post-embolization syndrome than TACE is. ${ }^{9,10}$

TARE is an intra-arterial therapy using ${ }^{90}$ Y-labeled microspheres (TheraSphere ${ }^{\circledR}$ or SIR-Spheres [Sirtex Medical, North Sydney, Australia]); the radiation induces extensive tumor necrosis. This therapy is increasingly used to treat HCC, especially in Western countries. ${ }^{17}$ Although there is no consensus regarding the optimal use of TARE, TARE is primarily considered for patients with locally advanced HCC and good liver function. ${ }^{9}$ Moreover, TARE is contra-indicated in patients with decompensated liver disease, a massive or infiltrative HCC replacing the entire liver, a severe comorbidity, or a high lung shunt fraction. Accordingly, prior to performing TARE, pretreatment simulation tests including hepatic angiography and a lung perfusion scan using ${ }^{99 \mathrm{~m}} \mathrm{Tc}-$ MAA should be performed to evaluate the vascular anatomy and lung shunt fraction. Patients with a lung shunting level that could result in a lung radiation dose $>30$ Gy per treatment or $>50$ Gy in total should not receive TheraSphere ${ }^{\circledR}$ therapy. When SIR-Spheres are used, 20\% lung shunting is the suggested limit; dose reduction is required when lung shunting is $10-20 \%$. The current patient had a single HCC that invaded the portal vein, with no extrahepatic metastasis, Child-Pugh score of 5, and lung shunt fraction of 5\%. He was thus an ideal candidate for TARE.

TARE and sorafenib resulted in comparable survival in patients with advanced HCC in two randomized trials: the SorAfenib versus Radioembolization in Advanced Hepatocellular carcinoma (SARAH) trial ${ }^{18}$ and the selective internal RT vs. sorafenib (SIRveNIB) trial. ${ }^{19}$ In the SARAH trial, the median overall survival was 8.0 and 9.9 months for the TARE and sorafenib groups, respectively $(P=0.18)$. In the SIRveNIB trial, the median overall survival was 10.0 and 8.8 months for the TARE and sorafenib groups, respectively $(P=0.36)$. However, the trials included patients with multiple/large tumors. Moreover, patients who had undergone previous treatment, such as those who had failed TACE, were also included. Therefore, the clinical outcomes of TARE might differ from those of sorafenib in patients with treatment-naive HCC that had low tumor burden (such as the current patient). Further studies in future are warranted to elucidate this clinical issue.

In conclusion, we described a case of a single HCC with segmental PVI that was successfully managed with TARE. Thus, TARE may be a useful alternative treatment option for patients with locally advanced HCC.

\section{Conflicts of Interest}

Dr. Yu SJ reports lecture fee from Bayer HealthCare Pharmaceuticals; Dr. Lee JH reports receiving lecture fee from GreenCross Cell, Daewoong Pharmaceuticals, and Gilead Korea; Dr. Kim YJ, research grants from Bristol-Myers Squibb, Roche, JW Creagene, Bukwang Pharmaceuticals, Handok Pharmaceuticals, Hanmi Pharmaceuticals, Yuhan Pharmaceuticals, Samjin Pharmaceuticals, and Pharmaking, and lecture fees from Bayer HealthCare Pharmaceuticals, Gilead Science, MSD Korea, Yuhan Pharmaceuticals, Samil Pharmaceuticals, CJ Pharmaceuticals, Bukwang Pharmaceuticals, and Handok Pharmaceuticals; Dr. Yoon JH, research grants from AstraZeneca, Bayer HealthCare Pharmaceuticals, Daewoong Pharmaceuticals, and Bukwang Pharmaceuticals. No other potential conflict of interest relevant to this article was reported.

\section{REFERENCES}

1. Bruix J, Sherman M; American Association for the Study of Liver Diseases. Management of hepatocellular carcinoma: an update. Hepatology 2011;53:1020-1022.

2. Bruix J, Cheng AL, Meinhardt G, Nakajima K, De Sanctis Y, Llovet J. Prognostic factors and predictors of sorafenib benefit in patients with hepatocellular carcinoma: analysis of two phase III studies. J Hepatol 2017;67:999-1008.

3. Cheng AL, Kang YK, Chen Z, Tsao CJ, Qin S, Kim JS, et al. Efficacy and safety of sorafenib in patients in the Asia-Pacific region with advanced hepatocellular carcinoma: a phase III randomised, double-blind, placebo-controlled trial. Lancet Oncol 2009;10:25-34.

4. Llovet JM, Ricci S, Mazzaferro V, Hilgard P, Gane E, Blanc JF, et al. Sorafenib in advanced hepatocellular carcinoma. N Engl J Med 2008;359:378-390. 
5. Yoon SM, Ryoo BY, Lee SJ, Kim JH, Shin JH, An JH, et al. Efficacy and safety of transarterial chemoembolization plus external beam radiotherapy vs sorafenib in hepatocellular carcinoma with macroscopic vascular invasion: a randomized clinical trial. JAMA Oncol 2018;4:661-669.

6. Sieghart W, Hucke F, Peck-Radosavljevic M. Transarterial chemoembolization: modalities, indication, and patient selection. J Hepatol 2015;62:1187-1195.

7. European Association for the Study of the Liver. EASL Clinical Practice Guidelines: management of hepatocellular carcinoma. J Hepatol 2018;69:182-236.

8. Marrero JA, Kulik LM, Sirlin CB, Zhu AX, Finn RS, Abecassis MM, et al. Diagnosis, staging, and management of hepatocellular Carcinoma: 2018 Practice Guidance by the American Association for the Study of Liver Diseases. Hepatology 2018;68:723-750.

9. Kim DY, Han KH. Transarterial chemoembolization versus transarterial radioembolization in hepatocellular carcinoma: optimization of selecting treatment modality. Hepatol Int 2016;10:883-892.

10. Kallini JR, Gabr A, Salem R, Lewandowski RJ. Transarterial radioembolization with yttrium-90 for the treatment of hepatocellular carcinoma. Adv Ther 2016;33:699-714.

11. Sangro B, Salem R. Transarterial chemoembolization and radioembolization. Semin Liver Dis 2014;34:435-443.

12. Moreno-Luna LE, Yang JD, Sanchez W, Paz-Fumagalli R, Harnois DM, Mettler TA, et al. Efficacy and safety of transarterial radioembolization versus chemoembolization in patients with hepatocellular carcinoma. Cardiovasc Intervent Radiol 2013;36:714-723.
13. Lencioni R, Llovet JM. Modified RECIST (mRECIST) assessment for hepatocellular carcinoma. Semin Liver Dis 2010;30:52-60.

14. Kudo M, Finn RS, Qin S, Han KH, Ikeda K, Piscaglia F, et al. Lenvatinib versus sorafenib in first-line treatment of patients with unresectable hepatocellular carcinoma: a randomised phase 3 noninferiority trial. Lancet 2018;391:1163-1173.

15. Shi J, Lai EC, Li N, Guo WX, Xue J, Lau WY, et al. Surgical treatment of hepatocellular carcinoma with portal vein tumor thrombus. Ann Surg Oncol 2010;17:2073-2080.

16. Pawlik TM, Poon RT, Abdalla EK, Zorzi D, Ikai I, Curley SA, et al. Critical appraisal of the clinical and pathologic predictors of survival after resection of large hepatocellular carcinoma. Arch Surg 2005;140:450-457; discussion 457-458.

17. Salem R, Gabr A, Riaz A, Mora R, Ali R, Abecassis M, et al. Institutional decision to adopt $Y 90$ as primary treatment for hepatocellular carcinoma informed by a 1,000-patient 15-year experience. Hepatology 2018;68:1429-1440.

18. Vilgrain V, Pereira $H$, Assenat $E$, Guiu B, Ilonca AD, Pageaux GP, et al. Efficacy and safety of selective internal radiotherapy with yttrium-90 resin microspheres compared with sorafenib in locally advanced and inoperable hepatocellular carcinoma (SARAH): an open-label randomised controlled phase 3 trial. Lancet Oncol 2017;18:1624-1636.

19. Chow PKH, Gandhi M, Tan SB, Khin MW, Khasbazar A, Ong J, et al. SIRveNIB: selective internal radiation therapy versus sorafenib in Asia-Pacific patients with hepatocellular carcinoma. J Clin Oncol 2018;36:1913-1921. 